\title{
Zur Praxis des Nachrichtenredakteurs beim Hörfunk
}

\author{
von Hanns Gorschenek
}

Die Massenkommunikationsmittel und die verschiedenen Bereiche der Kommunikationswissenschaft haben sich in den letzten Jahrzehnten stürmisch entwickelt. Der technische Fortschritt bei Hörfunk und Fernsehen und damit die nahezu lückenlose Einbeziehung des einzelnen wie aller relevanten Gruppen unserer Gesellschaft in den Kommunikationsprozeß hat die Verantwortlichen in Hörfunk und Fernsehen vor neue Aufgaben im Bereich des Mediums selbst, aber auch im Blick auf den Kreis der Rezipienten gestellt. Die Praktiker bedürfen - so stellt sich heute die Lage in viel stärkerem Maße als zur Zeit der Alleinherrschaft des "Dampfradios“, zur Zeit einer klar strukturierten und überschaubaren Gesellschaft, der Hilfe der Wissenschaft. Die Massenzahl ist Meßziffer im Kommunikationsprozeß geworden. Der Statistiker, der Soziologe, der Theologe, der Germanist, der Historiker, der Pädagoge, um nur einige beispielhaft herauszugreifen, sollten sich permanent aufgerufen fühlen, den Massenkommunikationsmitteln subsidiär zur Verfügung zu stehen. ${ }^{1}$

Das gilt insbesondere für die bei Hörfunk und Fernsehen tätigen NachrichtenJournalisten, die sich in der Tagesarbeit schnell verbrauchen, die ständig vor der Entscheidung stehen, wie weit sie als "gate keepers" die Schleusen für den Informationsfluß öffnen; sie stehen an ihrem Arbeitsplatz in einer permanenten Gewissensentscheidung und begeben sich in eine immerwährende Konfliktsituation. Die Verantwortlichen in Hörfunk und Fernsehen müssen das Gewissen zur Norm ihres Handelns machen, zu einer öffentlichen Gewissensentscheidung beitragen, die überhaupt erst einer öffentlichen Meinungsbildung vorausgeht. Wenn man unter Gewissen die Fähigkeit des Menschen versteht, das sittlich Gute als persönliche Forderung des Sittengesetzes zu erkennen und diese menschliche Fähigkeit auf das eigene Handeln anzuwenden, so ergibt sich auch für den publizistischen Bereich das $\mathrm{Ge}-$ wissen als höchste und letzte Instanz der Beurteilung menschlichen Handelns.

Der Vorgang der Kommunikation, den man vom Sittengesetz nicht abstrahieren kann, spitzt sich also auf das gewissenhafte Handeln des Korrespondenten, des Redakteurs im Massenkommunikationsmittel im wahrsten Sinne des Wortes zu: Bezogen auf den Teilaspekt der Vermittlung von Informationen im Hörfunk, also auf das Funktionieren einer Gemeinschaft, die rund um die Uhr Nachrichten von der Quelle bis zur Sendung erfaßt, formt und so schnell wie möglich den Rezipienten zukommen läßt. Diese Gemeinschaft der Redakteure und des übrigen Personals darf sich nicht als eine willkürliche Zusammenfassung von Individuen oder gar als eine in Einzelinteressen und Egoismen zerfallende lodkere Verbindung von Einzelwesen eines Berufsstandes verstehen, die sich lediglich um des Broterwerbs willen zufällig zusammengefunden haben. Wenn ein Nachrichtendienst funktionieren soll, muß sich eine Gemeinschaft in der Anonymität als Leistungsträger entwickeln, die in der Stille der Arbeit Vorbilder und Leitbilder hervorbringt sowie mit Idealismus und Hingabe an der politischen Informationsaufgabe arbeitet. Die Kommunikation

Hanns Gorschenek ist Leiter der Nachrichten-Zentralabteilung des Deutschlandfunk, Köln. 
ist also stark geprägt auch von einer individuellen und sozialen Komponente. Sie in das rechre Verhältnis zum publizistischen Auftrag zu bringen, ist Streben aller Rundfunkpublizisten und ihre Sorge zugleich.

Trotz der Fortentwicklung des Mediums Fernsehen zeigt die Praxis, daß der Hörfunk seine Aufgabe nicht verloren hat, sondern daß sein Wert als aktuelles Informationsinstrument laufend höher eingeschätzt wird. Der Hörfunk ist in der Lage, immerwährend zu informieren. Er schafft damit ein Nachrichten-Angebot, das in seiner Dichte unter den Kommunikationsmitteln einmalig ist. Was ergibt sich daraus?

Der Hörfunk ist technisch in der Lage, zu jeder Tageszeit und heute schon fast an jedem Ort das Informationsgebot der Stunde zu erfüllen. Dies erfordert aber klare und vor allem beständige Programm-Schemata, die nicht ohne wirklich zwingende Gründe und ohne Not über Bord geworfen werden sollten. Nachrichten müssen ihren festen Platz im Programm haben und behalten. Nachrichten-Sendezeiten sind ebensowenig manipulierfähig wie Nachrichten selbst. Für den Redakteur mag, ja sollte sogar, jede Nachrichtensendung zum Abenteuer werden, weil Nachrichten immer live gesendet werden und damit dem Wesen des Hörfunks am besten entsprechen. Nachrichten und Bandkonserven leben in Feindschaft. Niemals aber solltc der Hörer eine Nachrichtensendung als Abenteuer empfinden. Nachrichtensendungen erst aufsuchen $\mathrm{zu}$ müssen, beispielsweise auf Grund des Studiums von Programmzeitschriften, spricht gegen die Programmklarheit und Programmsystematik einer Anstalt. Der Nachrichtenarbeit unter Zeitdruck entspricht die Informationsabgabe ohne Zeitverlust, vor allem in den Morgenstunden, aber auch in den übrigen fernsehfreien Tageszeiten.

$\mathrm{Daß}$ der Fluß der Nachrichten zu bestimmten Tageszeiten angehalten werden muß, $\mathrm{da}$ ß der Hörfunk nicht immer ausschließlich mit dem Ereignis wandern kann, um nicht lückenhaft, sporadisch, ungezielt (bezogen auf den Hörerkreis) zu informieren, versteht sich von selbst und ergibt sich aus der Verantwortung denjenigen gegenüber, die nicht in der Lage sind, jede Nachrichtensendung, sondern vielleicht nur zwei oder drei Hauptnachrichtensendungen einer Anstalt am Tage zu hören.

An diesem Punkt des Zusammenraffens zu einer bestimmten Tageszeit für einen bestimmten Hörerkreis wird das Spannungsverhältnis zwischen Punktdarstellung, Aktualität und Nachrichtenauswahl, in dem sich der Redakteur befindet, besonders sichtbar. Hier kehrt sich nämlich das Leistungsprinzip hervor mit seiner ganzen Problematik für den Nachrichtendienst einer Rundfunkanstalt. Der Satz galt schon immer, daß der Nachrichten-Redakteur sein Geld in den letzten zehn Minuten vor jeder Sendung verdient. Aber vielleicht ist es zweckmäßig, die Arbeit des Nachrichten-Redakteurs einmal unter einem anderen Gesichtspunkt zu betrachten. In Wahrheit wird im herkömmlichen Sinne hier nicht gearbeitet, sondern geleistet. Kaum für einen anderen Beruf im Rundfunk gilt das einfache physikalische Gesetz wie für den des Nachrichten-Redakteurs, nämlich: Leistung ist Arbeit in Zeiteinheit. ${ }^{2}$ Denn der politisch Interessierte - und der Hörfunk hat das Glück, in sehr hoher Zahl politisch Interessierte anzusprechen - will auf Knopfdruck aktuell, allgemein verständlich, knapp und objektiv informiert werden. Weil das so ist, erfreuen sich bei mehr als 80 Prozent aller Hörfunkteilnehmer Nachrichten großer Beliebtheit.s Der Anspruch des Hörers ist für jeden Nachrichten-Redakteur eine unerhört große Verpflichtung, mit den entsprechenden Anforderungen an sein Gewissen, sein politisches Geschick und sein Sprachgefühl, immer gemessen am Auftrag des Gesetzgebers für die jeweilige Anstalt. 
Vielleicht sollte an diesem Punkt einmal vom modernen Medium Rundfunk weggeblendet werden, hin auf eine historische Betrachtung der Nachrichtenentwicklung und der Nachrichtenarbeit, um ermessen zu können, in welch schwieriger Tagesaufgabe der Redakteur heute bei der Nachrichtengebung im Rundfunk steht. Mit der praktischen Anwendung der drahtlosen Telegrafie trat in den Zwanziger Jahren des 20. Jahrhunderts neben die Presse ein neues Nachrichten-Medium: der Hörfunk. Der Weg von der Nachrichtenquelle zum Nachrichtenempfänger verkürzte sich enorm. Satz, Druck und Vertrieb der Zeitung wurden überholt durch das neue technische Medium. Es ergab sich eine natürliche Abgrenzung zwischen Zeitung und Rundfunk. Die Zeitung, die bis dahin Nachrichtenträger Nr. 1 war, mußte nach neuen Formen der Informationsabgabe suchen. Sie fand sie in der zusammenfassenden und wertenden Gesamtdarstellung, in der Vermittlung von Nachricht, Bericht und Kommentar, die zum "Aufmacher" wurde, in der Hintergrundwertung, in der Analyse und in der Dokumentation. Daraus ergab sich wiederum die Aufgabe des Hörfunks: Die aktuelle sachliche Kurzinformation, die jedoch die ergänzende Information durch Zeitung und Fernsehen nicht überflüssig, sondern wünschenswert macht.

Die Zeitung verlor ihr Nachrichten-Monopol durch die technische Entwicklung. Der Hörfunk öffnete im Bereich der Information neue Horizonte. Die Mängel einer „flüchtigen Aussage des modernen Zeitungssängers", wie vor einigen Jahren einmal ein Zeitungswissenschaftler die Hörfunknachricht genannt hatte, können durch Zuhilfenahme neuer Programm-Methoden ausgeglichen werden, nämlich durch häufig wiederkehrende und damit als Wiederholung verstandene Sendungen, um den Hörer von einer bestimmten Zeit soweit wie möglich unabhängig zu machen.

Nachricht ist die kürzeste Mitteilung eines Sachverhalts (nach Hagemann) ${ }^{4}$ und nach Dovifat die „Mitteilung über neue im Daseinskampf des einzelnen und der Gesellschaft auftauchende Tatsachen" ${ }^{\text {. }}$. Grimms Deutsches Wörterbuch definiert die Nachricht als eine "Mitteilung zum Darnachrichten" ${ }^{\text {" }}$, womit umschrieben ist, wie Dovifat in seiner soeben neu aufgelegten „Zeitungslehre“ sagt, daß die Nachricht für den Empfangenden von Wert und Nutzen sein muß. Aus allen Definitionen ergibt sich eindeutig, daß schon mit einem kräftigen ideologischen Hobel gearbeitet werden muß, um Nachrichten zu fälschen und zu manipulieren. Tut man dies, sind es eben keine Nachrichten mehr, sondern Wortbeiträge zur Agitation. Wenn Goebbels sagte: "Keine Nachricht ohne Tendenz ${ }^{\text {, }}$, so muß in der Nachrichtengebung in unseren Rundfunkanstalten der Grundsatz Geltung haben: Keine Nachricht mit Tendenz und keine Nachricht ohne gewissenhafte Prüfung des Sachverhaltes.

Nimmt der Nachrichten-Redakteur die wenigen Grundgesetze seiner Arbeit ernst - und es bleibt ihm gar nichts anderes übrig, wenn er nicht in kurzer Zeit scheitern will -, so geht er von Stunde zu Stunde einen dornenvollen und gefährlichen Weg. Er muß nicht nur Gehalt und Gewicht einer Tatsachenmitteilung in das rechte Verhältnis setzen zum öffentlichen Interesse und zum Daseinskampf des einzelnen in unserer Gesellschaft. Er muß auch die Durststrecke verantworten, die diese Tatsachenmitteilung vom Ausgangspunkt bis zum Empfänger mitmacht und soll dabei auch noch die verschiedenartig gelagerte Aufnahmebereitschaft seiner Hörer in Rechnung stellen. Am Nachrichtentisch muß immer wieder die Frage gestellt werden, wieviele geistige Relaisstationen eine Meldung bereits passiert haben mag, ehe sie beim Konsumenten ankommt. Der Redakteur muß die Kanäle kennen, durch die sich eine Meldung quält, er muß die vielen feinen Nachrichtenstränge unter Kontrolle halten, weil er nur dann richtig urteilen und so den Wert des Nachrichten- 
stoffes qualifizieren kann. Er muß auch wissen, aus welchen Quellen die Agenturen schöpfern, denn das Nachrichtenbild kann bei der Vielfalt der Quellen eingefärbt sein. Der Nachrichten-Redakteur muß also kritisch und immer mit größter Skepsis an den Stoff herangehen.

Wir wissen aus dem täglichen Umgang mit der Nachricht, daß es immer schwierig ist, Sendestoff, Sendezeit und Sendefolge in eine optimale Übereinstimmung zu bringen. Das Nebeneinander der Meldungen, wie es sich für den Zeitungsleser darstellt, redigiert und zum Ausdruck gebracht wird durch technisch-graphische Hilfsmittel, möglicherweise noch ergänzt durch ein Bild, wird beim Rundfunkhörer abgelöst durch das Nacheinander des Gebotenen, das zudem ausschließt, „nachgehört" zu werden. Daraus ergeben sich für das Gestalten und Formulieren einer Rundfunknachricht eiserne Gesetze.

Sie lassen sich zusammengefaßt so darstellen:

1. Der Nachrichtenkern, der wesentliche Inhalt einer Meldung, muß am Anfang stehen. Die Begriffe müssen logisch geordnet sein.

2. Die Sätze sollen, ebenso wie die Meldung selbst, kurz sein, um die Aufnahmefähigkeit des Hörers nicht zu überfordern.

3. Zahlen sind sparsam zu verwenden. Das beste Bild geben Verhältniszahlen.

4. Distanzieren von der Nachrichten-Quelle ist ein wesentliches Element sauberer Nachrichtenarbeit. Es hat den Vorteil, den Hörer an der Kritik des NachrichtenMaterials teilnehmen zu lassen.

5. Im Nachrichtenstil ist auf Bildwirkung zu achten, jede Hörhilfe anzubieten.

6. Die Nachricht, insbesondere die Rundfunknachricht, gleicht einer verderblichen Ware. Sie muß also, wenn sie auf den Markt kommt, rasch abgenommen und den Rezipienten zugeführt werden. Die Aktualität rückt angesichts der immer größer werdenden Zahl von Nachrichtendiensten in den Rundfunkanstalten mehr und mehr in den Vordergrund und bestimmt das Nachrichtengeschäft.

7. Die gewissenhafte Prüfung des Wahrheitsgehaltes darf dabei nicht zu kurz kommen. Die Recherche von der Redaktion her muß wieder verstärkt werden.

In jeder Redaktion wird um die Einhaltung dieser Gesetze immer wieder gerungen werden müssen, wenn es nicht zu einer Art Nachrichtenanarchie im Hörfunk kommen soll. Ansätze dazu sind dort zu verspüren, wo man es nicht mehr ganz so ernst nimmt mit der scharfen Trennung von Tatsache und Meinung in einer Nachricht, wo man sich im Blick auf den "Aufmacher" der Zeitung zum sogenannten Leadsatz hinreißen läßt, der meist von der harten Tatsache wegführt und ins Verschwommene, erläuternde Zusammenfassen abgleitet und damit oft die Meinungsbildung vorwegnimmt, statt harte Tatsachen zur eigenen Meinungsbildung zu vermitteln.

Denjenigen, denen die Rundfunknachricht heute zu abstrakt, zu wesenlos, zu introvertiert, zu wenig glatt, allzustark tatsachenbezogen, zu wenig gut „verpackt“ zu sein scheint, möchte ich eigentlich nur das in Erinnerung rufen, was der Chefredakteur des Drahtlosen Dienstes, Räuscher, vor fast vierzig Jahren in Wort und Schrift zur akustischen Nachrichtenvermittlung gesagt hat. ${ }^{7}$ Seine Regeln sind heute so aktuell wie damals. Zusammengefaßt und adaptiert möchte ich sie so wiedergeben: 
1. Schreibe Nachrichten so, wie du sie als Hörer wünschen würdest!

2. Die Sprache ist Verständigungsmittel, nicht Preisrätsel!

3. Unsere Arbeit wird rascher Klang, nicht bleibendes Schriftbild!

4. Der Hörer ist nicht Leser, nicht Redakteur, nicht Fachmann!

5. Der Hörer pfeift auf Amtsdeutsch und Berufsjargon!

6. Der Hörer ist müde und will leicht verstehen!

7. Der Hörer hat für die Aufnahme der Nachricht nur die Ohren zur Verfügung!

8. Die Hörerschaft setzt sich zusammen aus allen Stämmen, Konfessionen, Ständen, Parteien und Bildungsschichten!

9. In der Auffassungskraft urteile nach dem naivsten Hörer!

10. Wer am Wortlaut der Agentur klebt, ist Briefträger, nicht Redakteur!

11. Nur was man sich selbst völlig klargemacht hat, kann man einfach und klar wiedergeben!

Von diesen Grundregeln ausgehend, gibt Räuscher dann noch einige Ratschläge für den Nachrichtenstil. Er wollte die Nachricht vom Substantiv und der Substantivierung befreien, zugunsten des Verbs - wir haben es leider heute immer noch nicht geschafft! Er wollte mit Recht die logische Zuordnung der Reihenfolge der Begriffe, er verlangte die Bildwirkung und warnte zugleich vor falschen Bildern, er plädierte für kurze Sätze, wollte jede Hörhilfe aufgeboten wissen. Für uns heute besonders interessant und aktuell, meine ich, ist Räuschers Hinweis: „Vorsicht bei abgetretenen Gebieten, damit nicht der Anschein nationaler Preisgabe erweckt werde!"

Die Technik hat der Nachricht im Hörfunk eine Chance gegeben. Die Verantwortlichen in den Massenmedien sollten sie begreifen und daraus die Konsequenzen ziehen, auch für die Arbeit im kirchlichen Bereich. Die Kirche selbst hat eine „Nachricht" auszurichten, sagte Johann Baptist Metz bei einer Tagung der Katholischen Rundfunk- und Fernseharbeit in Hamburg. ${ }^{8}$ Sie bedient sich des Mediums Rundfunk und unterwirft sich damit auch den dort geltenden Gesetzen. Die Zahl der Nachrichten-Quellen kann klein oder groß sein. Auf die Seriosität dieser Quellen ist zu achten. Die Auswahl der Nachrichten geschieht in erster Linie im Blick auf den gesetzlichen Auftrag der Anstalt, gemessen an der Aktualität, sich spiegelnd im Pluralismus unserer Gesellschaft, nicht zuletzt aber auch unter Würdigung dessen, wie Dovifat es formulierte, daß die Nachricht für den Empfänger von Wert und Nutzen sein muß.

Wenn der Hörfunk seiner Aufgabe gerecht werden will, muß er dafür sorgen, daß die Kontinuität des Gebotenen unter Berücksichtigung auch für den „Zufallshörer erkenntlich wird, daß der "gate keeper" die Schleuse im Fluß der Nachrichten also immer soweit offen hält, wie es das Verständnis für Zusammenhänge erforderlich macht. ${ }^{9}$ Der Hörfunk muß sich immer wieder auf seine aktuelle InformationsVerpflichtung besinnen und Zäsuren setzen bei der Weitergabe des Nachrichtenmaterials. Er muß sich gegenüber der Zeitungsnachricht durch die knappste Form der Darstellung abgrenzen und die Ergänzung zur Nachricht in Form des Kommentars, des Berichts, der Analyse, des Interviews geradezu herausfordern. Die Nachricht bedarf der Ergänzung durch die Hintergrunddarstellung in Funk, Fernsehen und Zeitung. Wer dies will, plädiert zugleich für die saubere Trennung von Nachricht und Meinung. 


\section{Anmerkungen:}

1. Vgl. Becker-Siegel (Hrsg.), Dynamik der Kommunikation (Rundfunk und Fernsehen im Blick der Kirche, Beiheft 8), Frankfurt 1968, passim.

2. Vgl. Hanns Gorschenek, Nachrichten im Hörfunk (Deutschlandfunk 3/67), Köln 1967, S. 27.

3. Vgl. ARD (Hrsg.), Rundfunkanstalten und Tageszeitungen - Eine Materialsammlung (Dokumentation 4), Frankfurt 1966, S. 16.

4. Walter Hagemann, Die Zeitung als Organismus, Ein Leitfaden, Heidelberg 1950, S. 49.

5. Emil Dovifat, Zeitungslehre I, Berlin ${ }^{4} 1962$, S. 54.

6. Jacob und Wilhelm Grimm, Deutsches Wörterbuch, Bd. VII, Leipzig 1889, Spalte 103 (Bearbeitung Lexner).

7. Josef Räuscher, Der gesprochene Nadrichtendienst, in: Rufer und Hörer, Berlin 1927, Heft 5, S. 33.

8. Johann Baptist Metz, Verkündigung und Gesellschaft, in: Dynamik der Kommunikation (Rundfunk und Fernsehen im Blick der Kirche, Beiheft 8), Frankfurt 1968, S. 15.

9. Zur Funktion der "Gatekeepers" im publizistischen Prozeß vgl. Henk Prakke u. a., Kommunikation der Gesellschaft, Einführung in die funktionale Publizistik, Münster 1968, S. $77 \mathrm{ff}$.

\section{S U M M A R Y}

Even in the age of television the old radio sound broadcast hasn't lost its importance. As a means for communicating "hot" information it is still unbeatable today. Practically speaking, radio is the medium losing least time on giving the news of any other means of mass-communication. Herein lies the power and the responsiblity of the news editor working in a radio station: He has to check the incoming information in the shortest possible time and has to communicate it as objectively as possible. Besides the rules effective in every mass-medium, the editor of a sound broadcast news service has to bear in mind some specific ones, such as - compared with a newspaper - being as short as possible but stimulating the desire of the reader to get more detailed and deeper information through other media.

\section{RES U MEN}

En la época de la Televisión, y a pesar de las opiniones contrarias, la Radio no ha perdido su importancia. Hasta el presente, como instrumento de información, no ha sido superada por ningún otro medio. Continúa siendo el medio de comunicación que propaga las noticias con la máxima rapidez. Este es su punto fuerte, y también la dificultad, a la que se hallan sometidos los agentes de noticias: el de examinarlas concienciosamente en el mínimo espacio de tiempo, y el de transmitirlas sin la intención de crear ninguna tendencia, en el campo del pensamiento, a los lectores $u$ oyentes. Junto a las normas fundamentales que todo periodista debe respetar por principio, el redactor de noticias para la Radio debe observar otras muchas normas específicas de la publicidad. La Radio cumple su cometido a la perfección, cuando se distingue de los periódicos por la brevedad de las noticias, despertando con ello en los oyentes el deseo de análisis y opiniones complementarias. 